\title{
Identificación y caracterización de mudas de transporte, procesos, movimientos y tiempos de espera en nueve pymes manufactureras incorporando la perspectiva del nivel operativo
}

\author{
Identifying and characterizing of wastes (Muda) in transportation, processes, \\ movements, and waiting time, in nine manufacturing SMEs incorporating the \\ perspective of the operational level
}

\author{
Jorge Pérez Rave ${ }^{1} \quad$ Daniel La Rotta $^{1} \quad$ Katherine Sánchez $^{1} \quad$ Yiseth Madera $^{1}$ \\ Guillermo Restrepo $^{1} \quad$ Mayra Rodríguez $^{1}$ Johan Vanegas $^{1} \quad$ Carlos Parra $^{1}$ \\ Recibido 24 de junio de 2010, aceptado 7 de diciembre de 2011 \\ Received: June 24, 2010 Accepted: December 7, 2011
}

\begin{abstract}
RESUMEN
Se identifica y se caracteriza en términos de: cantidad, tipo, localización y manifestación, los transportes, tiempos de espera, movimientos y procesos innecesarios en nueve pymes manufactureras de la ciudad de Medellín. El procedimiento constó de las etapas: selección de procesos a estudiar (uno por empresa), capacitación al personal operativo ( 7 mudas, $5 \mathrm{~S}$ y gerencia visual), sesión en profundidad con administración de formulario de identificación del muda, verificación en el gemba, y resultados y análisis. Consolidando los hallazgos para las nueve empresas, 151 trabajadores fueron capacitados, los cuales contribuyeron a identificar 1.085 actividades que no aportan valor al cliente, con un promedio de 121 por proceso-empresa, la mayoría fueron transportes (31\%) y movimientos innecesarios (29\%). Se brindan elementos metodológicos que facilitan la comunicación entre el nivel operativo y los demás, así como la capacitación y la participación activa del mismo, posibilitando identificar oportunidades de mejora para las empresas. Se ofrece información de interés para la academia, el gobierno, los centros de desarrollo tecnológico y el sector productivo.
\end{abstract}

Palabras clave: Pymes, mudas de manufactura, Lean manufacturing, mejora continua.

\section{ABSTRACT}

Is identified and characterized in terms of: quantity, type, location and expression, transportation, waiting times, movements, and unnecessary processes, in nine manufacturing SMEs in the city of Medellin. The procedure consisted of the stages: selection of processes to be studied (one per company), operating personnel training (7 Wastes (Muda), $5 S$ and visual management), deep session with management of the Muda identification form, verifying in the shop floor (Gemba), results and analysis. Consolidating the findings for the nine companies, 151 workers were trained, which helped to identify 1.085 activities that do not add value to the customer, with an average of 121 per processcompany, most were transportation (31\%) and unnecessary movements (29\%). Methodological elements are provided to facilitate communication between the operational level and others, as well as training and active participation of the same, making it possible to identify opportunities for business improvement. It offers information of interest to academia, government, technology development centers and the productive sector.

Keywords. SMEs, manufacturing wastes, Lean manufacturing, continuous improvement.

1 Grupo de investigación Gestión de la Calidad, Departamento de Ingeniería Industrial. Universidad de Antioquia. Cl1 67 № 53·108, Bloque 21, oficina 404. Medellín, Colombia. E-mail: ejipr056@udea.edu.co; investigagcalidad@gmail.com 


\section{INTRODUCCIÓN}

Las pymes manufactureras se ven inmersas en cambios derivados del entorno globalizado, los cuales exigen incesantes esfuerzos por incrementar la calidad y la productividad, y por reducir los costos y los tiempos de entrega a fin de lograr posiciones ventajosas en el mercado.

En Colombia, por ejemplo, estas empresas representan más del $90 \%$ de las unidades económicas y concentran cerca del $60 \%$ del empleo [1-2].

A pesar de la relevancia de las pymes, hay fuentes que brindan cifras entre 60\%-90\% para la tasa de desaparición de estas empresas, sin siquiera haber superado el quinto año de operación [3-4]. Además, las pymes enfrentan varios obstáculos para su desarrollo, entre ellos: problemas de acceso a los mercados, barreras tecnológicas y dificultades para obtener créditos. En términos de operación interna, presentan las siguientes falencias en sus dimensiones estructurales [5]:

- Sistema de gestión: desconocimiento de las necesidades de los clientes y de las tendencias del entorno, ausencia de planeación de la estrategia y de su despliegue, se maneja un enfoque reactivo en el actuar ("apaga incendios").

- Procesos: se desconoce la dimensión de los procesos y las relaciones entre ellos. Existe gran número de actividades sin valor para el cliente. Presentan niveles de productividad inferiores a estándares internacionales.

- Talento humano: no hay conciencia del papel que juega el trabajador en la producción y eficiencia de la empresa, poca o nula capacitación, escasas condiciones para que el trabajador libere el potencial humano.

- Información: ausencia de sistemas de indicadores, confiables, para: definir objetivos y metas, monitorear el logro de los mismos, y tomar decisiones con base en datos y hechos.

Entre las alternativas existentes para apoyar a las pymes en la superación de dichas falencias, sobre todo en la parte de procesos y la humana, Lean Manufacturing $(L M)$ posibilita, entre otros: la satisfacción de los clientes, el incremento del valor agregado a los productos, la participación de los empleados en los procesos de mejoramiento, y el aumento de ganancias para los inversionistas. A pesar de estos beneficios, dado el entorno dinámico y cambiante en el que deben moverse las empresas, viene la necesidad de que $L M$ permanezca en continua evolución para el ámbito de las pymes, motivando la generación de nuevas herramientas de gestión orientadas a reducir el muda de manufactura (aquellas actividades que no agregan valor para el cliente).

Bajo este contexto, el grupo de investigación Gestión de la Calidad, del Departamento de Ingeniería Industrial de la Universidad de Antioquia, desde el año 2004 viene integrando esfuerzos alrededor de las pymes. El presente artículo es derivado de uno de los proyectos más recientes del citado grupo de investigación, y se enmarca en la línea de mejoramiento paso a paso. Se delimitó a estudios de caso abordando el muda en nueve pymes manufactureras.

Si bien existen siete tipos de muda, el estudio se enfocó en aquellos directamente asociados al contenido del trabajo, constituyéndose en operaciones que ejecuta el trabajador operativo. Así, delimitando el muda a este tipo de tareas, se pretende aportar respuestas a las siguientes preguntas de investigación: ¿Cuáles son los transportes, procesos, movimientos y tiempos de espera que, desde la perspectiva del trabajador operativo, hacen presencia en las pymes involucradas en el proyecto? y ¿qué características presentan dichos desperdicios, en términos de: cantidad, tipo, localización y manifestación (en qué consisten)?

El presente artículo comparte información útil para empresarios, docentes, estudiantes e investigadores, en términos del estudio de las actividades, sin valor para el consumidor, que hacen presencia en las pymes intervenidas, y más aún, de una fuente tan relevante como es el personal operativo.

En el segundo apartado se expone un marco conceptual, fundamental para la comprensión del manuscrito por parte de público no especializado. El tercer apartado presenta la revisión literaria, el cuarto ofrece el procedimiento seguido para responder las preguntas de investigación, el quinto trata los resultados, el sexto apartado presenta la discusión, le siguen las conclusiones y se finaliza con los agradecimientos. 


\section{MARCO CONCEPTUAL}

\section{Lean manufacturing (LM)}

En la primera mitad del siglo pasado dos guerras mundiales destruyeron parte de la infraestructura física y económica de Europa y Japón, dejando cantidad de víctimas. Se notó supremacía comercial de algunos países al poder vender grandes cantidades de mercancía a las naciones más afectadas, siendo fundamental la producción en masa, bajo la filosofía del "empuje", la cual representa: producir primero y vender después [6]. En ello, los altos inventarios y defectos no eran problemas centrales.

Cuando comienzan a levantarse los países afectados, se fragmenta la demanda, se elevan los precios de los recursos (como el petróleo), se mueve la competencia en los mercados y surgen otras estrategias de gestión de la producción. Es precisamente en Japón, con grandes limitaciones de recursos naturales y de espacios, donde se inaugurará una guerra al desperdicio con las consignas de "cero defectos, cero inventarios, cero demoras y cero desprecio por las personas". La filosofía de la producción pasa del "empuje" al "halar": primero se vende, luego se produce; esto significa que el flujo se ajusta a la demanda, posibilitando reducir los inventarios mediante el sistema "Justo a tiempo".

Sumado a ello, cobra importancia el trabajo en equipo para controlar la calidad y el proceso, se diseñan dispositivos "a prueba de errores" y se fortalece la necesidad del mejoramiento continuo en toda persona, momento y lugar. Esta premisa, junto a la integración de técnicas y herramientas para reducir los desperdicios de manufactura ( $m u d a$, en japonés), de una manera sistémica y humana, dio lugar al Sistema de Producción Toyota (TPS), creando en Occidente la filosofía del Lean Manufacturing.

\section{Desperdicios de manufactura (muda)}

Representan todo aquello que no es la cantidad mínima de equipos, materiales, insumos, piezas, locaciones y tiempos de máquinas o de trabajadores, que resultan absolutamente esenciales para añadir valor al producto o servicio [7]. A continuación se describen los siete tipos en que se clasifican los desperdicios de manufactura [8-9]:

Sobreproducción: es la madre de los desperdicios y depende en su mayoría de los responsables de la toma de decisiones estratégicas y tácticas. $\mathrm{La}$ sobreproducción se refiere a programar la utilización de recursos en un momento y en cantidades que realmente no se requieren para satisfacer el consumidor.

Inventarios: su sostenimiento prologando y excesivo es perjudicial. Se divide en: materia prima, producto en proceso y terminado, genera costos de almacenaje y manipulación, propicia obsolescencia, defectos y sensación de poca capacidad.

Transporte: se caracteriza por el desplazamiento de elementos, bien sea materiales, producto en proceso/ terminado, personas o herramientas. Durante ese lapso de tiempo la organización no está modificando características, de forma o fondo, del producto, por las cuales el cliente esté dispuesto a pagar.

Movimientos innecesarios: a veces son poco efectivos los diseños de puestos, que obligan al colaborador a efectuar movimientos que fuerzan los desplazamientos normales de las extremidades, obligándolos a agacharse para recoger un insumo o herramienta, inclinarse, estirarse forzosamente, entre otras, colocando en riesgo la salud y generando un entorno poco productivo. También, el desorden del puesto de trabajo lleva a este desperdicio, por ejemplo, cuando se mezclan los elementos necesarios con los innecesarios, es preciso realizar varias manipulaciones para encontrar lo que se requiere.

Tiempos de espera: representa el evento de que los recursos cuya misión, en un momento dado, es no detenerse, se encuentran en dicho estado. Por ejemplo, cuando en un centro de trabajo sale una unidad de producto y debe esperar un tiempo para que sea procesada. Esto genera costos innecesarios y puede posibilitar pereza en la persona ociosa y bajo rendimiento cuando se vuelva a ocupar. Las esperas se deben a carente nivelación de cargas de trabajo, fallas en la programación o en equipos, ausencia de $5 \mathrm{~S}$, entre otros.

Procesos innecesarios: comprende actividades que existen por el diseño de procesos poco robustos e ineficientes, o por presencia de defectos. Por ejemplo, suponga el evento de reprocesar una pieza, ante control ineficiente a la calidad del proveedor. 
Defectos: se refiere a aceptar, producir o enviar productos que no cumplen con las especificaciones del cliente, bien sea interno o externo. Genera, a su vez, procesos innecesarios. Se le atribuyen directamente los costos de no calidad, hace perder tiempo valioso y puede afectar no solo la parte productiva o la económica, sino la misma satisfacción del cliente interno y externo.

No todos los desperdicios pueden eliminarse, pero con su reducción es posible impactar favorablemente el sistema productivo. Entre las principales herramientas para reducir los desperdicios, en las que se basa el trabajo realizado, están: $5 \mathrm{~S}$, gerencia visual y Cap-Do. A continuación se comentan:

5S: filosofía japonesa que integra sentido común, capacitación, autonomía en el trabajo y búsqueda de autorrealización del individuo, para generar un entorno donde se adopte la disciplina del orden, limpieza, seguridad, productividad y respeto por los estándares. Sus pasos: Seiri (Clasificar), Seiton (Ordenar), Seiso (Limpiar), Seiketsu (Estandarizar) y Shitsuke (Disciplina). $5 \mathrm{~S}$ va de la mano de gerencia visual, que mediante el uso de símbolos permite visualizar el estado de los procesos en comparación con el estándar y gestionar la comunicación en la empresa, dándose a conocer la estrategia general, registros de desempeño e incluso sugerencias del personal [10].

El ciclo CAP-Do (Check, Analize, Plan, Do) es una adaptación del PHVA (Planear, Hacer, Verificar y Actuar), siendo útil en solución de problemas, ya que inicia con la verificación del objeto de estudio, a fin de conocerlo, desplegando como segundo paso el análisis, luego la planeación y finalmente la ejecución [11].

\section{Metodología de identificación y reducción del muda desde la perspectiva del nivel operativo} Surge en el grupo de investigación Gestión de la Calidad, especialmente para pymes. Su despliegue consume pocos recursos y tiempo, y se compone de tres etapas: capacitar, identificar y reducir, las cuales giran en torno al nivel operativo y a la reducción de los desperdicios de manufactura, formando un triángulo de mejora. El objetivo de la etapa "capacitar" es generar confianza y dotar a los trabajadores de elementos básicos, actitudinales, conceptuales y procedimentales, para que estén en capacidad de identificar fácil y rápidamente cuándo una determinada actividad de producción aporta o no valor al cliente.

"Identificar" pretende conocer los principales desperdicios de manufactura en función de: ¿cuántos son?, ¿cuáles son?, ¿cómo son? y ¿dónde están?, lo cual se realiza a partir de la voz de las personas más conocedoras de los detalles del trabajo: el personal operativo.

"Reducir" se enfoca en responder a los interrogantes: ¿dónde, cómo y cuándo actuar? para potenciar el mejoramiento del proceso. La materialización de esta etapa se realiza a través de tres momentos: priorización, definición e implementación de acciones y mejora continua.

Desde una perspectiva macro, este manuscrito está delimitado a las dos primeras etapas: capacitar e identificar. Al interior de cada una de las etapas se hace girar el ciclo Cap-Do: chequear, analizar, planear y hacer.

\section{REVISIÓN DE LITERATURA}

La consulta fue realizada el 29 de diciembre de 2010 por medio de Scopus, uno de los buscadores científicos más posicionados de la Web [12]. Se extrajeron los 21 trabajos más citados entre 20062010 para cada uno de los siguientes términos, incluidos en el título de los documentos: "Toyota Production System" (11 documentos, los dos últimos empatados con 5 citaciones) y "Lean Manufacturing" (10 documentos). A continuación se describe brevemente cada trabajo:

En [13] se aplican principios del Sistema de Producción Toyota (TPS) en el ámbito de un laboratorio clínico; específicamente rediseñaron los procesos de la fase preanalítica, obteniendo una disminución del tiempo de respuesta, pasando de 29 a 19 minutos, posibilitando la meta de entrega del $80 \%$ de los resultados de las pruebas químicas en menos de una hora durante 11 meses consecutivos.

También en el ámbito de la salud, en [14] analizan si el uso de métodos del TPS permiten mejorar la calidad de la prueba de Papanicolau. Impactando en el diseño del flujo de las operaciones para mejorar la calidad durante el proceso, reducir ineficiencias 
y costos, los autores comprueban, mediante datos experimentales y de control, que el uso de métodos del TPS mejora la calidad de la prueba sometida a consideración.

Por su parte, como contraposición a las ventajas y ejemplo del éxito comúnmente posicionado sobre el TSP, haciendo alusión al "lado oscuro del lean", en [15] se hace una crítica sobre la efectividad del mismo, basada en la experiencia como empleado de la firma durante tres años; en el artículo resalta, entre otros, fenómenos de opresión de los trabajadores, riesgos para la salud, deficientes mecanismos para la innovación y un costo humano elevado para cumplir con las normas establecidas.

En [15] se analizan los efectos del apoyo a la gestión y a la comunicación organizacional durante la implementación del LM, a través de un estudio de caso, en una empresa de fabricación electrónica en la que recolectaron datos durante tres meses. Concluyen sobre la relevancia de estas dos variables para la aplicación del LM, infiriéndose que la alta dirección debe evidenciar compromiso, ejercer liderazgo y comunicar los cambios a todos los actores de la organización. Sugieren un estudio en otras organizaciones con mayor historial en términos del LM, ya que la abordada se encontraba en las primeras etapas de implementación de dicha filosofía.

En [16] se realiza un estudio para aumentar la productividad en una planta de ensamble de automóviles y satisfacer la demanda, a través del desarrollo de mejores prácticas para las líneas de soldadura de operación robótica. Para ello, la gestión de restricciones fue integrada con el LM; la primera para identificar los centros más críticos que limitan la capacidad del sistema y la segunda para descubrir, en dichos centros restricción, los desperdicios que los caracterizan.

En [17] se resalta el valor del LM para lograr un flujo continuo de producción, mayor flexibilidad y niveles de desperdicios mínimos. Manifiestan que los principios del LM han sido más aplicados en algunos componentes de la cadena de valor de los productos madereros secundarios (Ej: función, torneado, ensamble y acabado) que en la misma producción en bruto. A través de datos de una encuesta nacional de plantas secundarias de procesamiento de maderas, los autores encuentran hallazgos de desempeño que corroboran los beneficios para aquellas organizaciones que han implementado LM, entre los que se destaca la reducción del Lead Time. Además, exponen algunas dificultades para la implementación del LM en la producción en bruto, asociadas a: medición del desempeño, restricciones de maquinaria e incapacidad para controlar la producción fuera de las especificaciones.

En [18] se ofrece la experiencia de aplicar conceptos del LM en el descubrimiento de fármacos, impactando favorablemente en la oportunidad del proceso de síntesis en paralelo. En [19] exploran la introducción del LM en el ámbito farmacéutico, comparando además este sistema con las recientes buenas prácticas de manufactura en dicho sector. Al final, hacen hincapié en los retos que se derivan para la implementación del LM, enmarcado en el término Lean Pharma, mediante el análisis de las cuatro reglas expuestas por [20]: trabajo estandarizado, directa comunicación cliente-proveedor en toda la cadena de valor, flujo simple y acciones de mejora con soporte científico.

En [21] se resalta la pertinencia mundial del TPS y se discute sobre reglas, algunas con fórmulas matemáticas para la implementación del mismo. Estas se asocian, entre otros a: organización del trabajo bajo células de fabricación, alineación de la demanda con la producción mediante la sincronía entre el Takt time y el tiempo de ciclo, dispositivos Kanban para el control de inventarios y estandarización de puestos de trabajo.

El estudio de [22] se debe a la aplicación de principios del TPS en Virginia Mason Medical Center (VMMC); en dicha entidad el TPS se adapta mediante la filosofía de Alerta de Seguridad del Paciente (PSA en inglés), lo cual se asemeja al paro de una línea de producción en el momento en que un ocurra un problema y no reactivarla hasta que éste sea resuelto. Dicho fenómeno, en este caso, corresponde a que si la práctica o conducta de un empleado pueda generar algún daño al paciente, debe inmediatamente ser interrumpida hasta que el problema sea resuelto. Esta filosofía estuvo soportada por el compromiso de la alta dirección de la entidad, por la declaración de una política institucional, por recursos y por una línea telefónica abierta las 24 horas, siendo la comunicación un factor clave de éxito para el sostenimiento de la estrategia. Esta filosofía ha contribuido a la identificación de constantes oportunidades de mejoramiento que 
compromete a personal de todo nivel jerárquico, así como a una mayor seguridad para el paciente, eficiencia en la respuesta a los problemas y más participación de la gente.

En [23] se profundiza sobre el ADN asociado al TPS, conformado por: visión, principios, herramientas y organización de aprendizaje. Comenta sobre una serie de presuposiciones, las cuales se relacionan, entre otros, con: alistamiento de la producción, control estratégico de los inventarios y explotación de herramientas de ingeniería industrial, colocando esta disciplina como la clave para comprender el TPS. Por su parte, en [24] se presenta una recontextualización del TSP haciendo énfasis en: el creciente interés occidental por este sistema, la preocupación por las tecnologías de la información como soporte en los sistemas de producción, el diseño de puestos de trabajo, y la motivación de los trabajadores.

En [25] examinan el proceso de adaptación del TSP por parte de Hyundai Motor Company, destacando que fue realizado de manera evolutiva, no limitándose a como fue implementado en Toyota, sino considerando las particularidades de la compañía Hyundai. De manera general, el caso releva que el proceso de adopción del TPS amerita un complejo proceso evolutivo de aprendizaje organizacional, que no debe considerar una copia exacta, sino una interpretación y adaptación del mismo a las características particulares del campo de implementación, tanto internas como externas, y a su misma idiosincrasia, manifestando que comúnmente se pasa por alto en la literatura existente. Esto infiere la posibilidad de diferentes caminos para implantar la filosofía del TPS.

Por otra parte, en [26] se destacan los beneficios atribuidos al TSP para empresas con productos de alto volumen y con especificaciones debidamente estandarizadas, logrando reducciones de costos, mejora de la calidad y reducción de los tiempos de entrega; pero además, resaltan la importancia del TSP para cualquier tipo de empresa, dejando de verlo como un conjunto de herramientas específicas técnicas o un conjunto de "pastillas individuales", para en su lugar, comprenderlo como una filosofía que reúne un conjunto de principios interrelacionados de gestión, propicios para el aprendizaje organizacional. A modo de evidencia, exponen un estudio de caso de una firma de bajo volumen de productos y altamente personalizados, que emplea el TSP para comprender de mejor manera su proceso de elaboración de tejas arcilla, empoderar a sus empleados y obtener mayores ganancias.

En [27] presentan un nuevo método que incorpora la robótica en el TPS, en busca de una calidad más uniforme a nivel mundial; esta incorporación junto con la simulación computacional y la ingeniería digital, entre otras, propician la renovación del ambiente de trabajo, una alta confiabilidad del sistema, la educación de los operarios y el establecimiento de sistemas inteligentes de control de calidad; todo ello enmarcado en un TSP avanzado que posibilita también la reducción de costos, el aumento de la productividad y la empleabilidad del personal. Manifiestan además, que a futuro los talleres de producción tenderán a migrar hacia este tipo de elementos. En la demostración del trabajo en Toyota, se logra una reducción del 50\% de los costes de producción, obteniendo una alta productividad y aseguramiento de la calidad.

[28] se enfoca en el TSP bajo una perspectiva europea, a fin de brindar una mayor comprensión a las condiciones sociales y organizacionales que hacen viable dicho sistema. Así, enfatiza en la organización del trabajo, la configuración del mercado y la infraestructura de las plantas europeas de Toyota, proponiendo al final un enfoque alterno centrado en la contextualización y la acción humana.

En [29] analizan a través de la simulación, el comportamiento antes-después de implementar principios del Lean Manufacturing (LM) en una acería, con mayor énfasis en el Value Stream Map (VSM). Bajo los escenarios descritos ilustran a los directivos de la empresa los beneficios del LM en términos de la disminución del Lead Time y del inventario de producto en proceso.

En [30] se enfocan en el VSM, pero a pesar de que destacan su popularidad creciente, comentan además sus limitantes a la hora de representar el comportamiento dinámico de los procesos; para superar esta falencia presentan un método de modelado que incorpora el VMS con objetos de simulación específicos para esta herramienta, posibilitando la generación rápida y automática de los escenarios actual y futuro. Finalmente aplican el método de modelado a una empresa real, analizando tres escenarios futuros y las consecuencias de 
principios del LM, pasando del enfoque Push al Pull. Su vez, en [31] tratan el VSM como una de las principales herramientas para implementar algunos de los principios del LM, posibilitando la identificación de desperdicios en los procesos y oportunidades de mejora. Entre los beneficios con esta herramienta destacan: reducción del Lead Time, tiempo de ciclo y niveles de inventario. Como caso de éxito el trabajo describe la aplicación del VSM en una empresa manufacturera pequeña.

En [32] aplican técnicas del LM en un departamento de emergencias de salud, con el objetivo evaluar si la adopción de principios LM en este tipo de servicios genera resultados satisfactorios en el valor agregado en la atención de las emergencias. Las etapas seguidas fueron: capacitación del personal en LM, observación del servicio, análisis del flujo de pacientes, rediseño de los procesos, análisis de los nuevos procesos e implementación. Concluyen que el LM en dicho ámbito de aplicación mejoró la satisfacción de los pacientes y aumentó el valor agregado del servicio, mediante la adopción de pequeños cambios que incluyeron la participación activa de los empleados. Proponen que las organizaciones deben centrarse en los principios básicos del LM, en lugar de imitar cambios realizados en otras firmas.

En [33] también hace énfasis en el auge del LM en las organizaciones empresariales, bien sea motivadas por cuenta propia o por exigencia de sus clientes. Este trabajo consiste en un estudio de caso realizado en una PYME manufacturera, que se enfrenta a una decisión multicriterio para determinar las prácticas, herramientas y técnicas de producción que más le convienen. Luego de aplicar el método "proceso analítico en red" (ANP en inglés), concluyen que la mejor alternativa de decisión para la empresa, en comparación con los sistemas de producción alternativos, es la adopción del LM. Destacan la novedad de la aplicación del ANP en el ámbito del LM.

\section{METODOLOGÍA}

Se trata de nueve estudios de caso, en los cuales se siguió el procedimiento expuesto en la Figura 1.

La primera sesión se enfocó en la invitación a las empresas para que participaran en el proyecto.
Para ello, a partir de diversas bases de datos se contactaron pymes manufactureras del Valle de Aburrá. Posteriormente se les expuso el proyecto y considerando: sector, clasificación de la empresa, interés manifiesto en participar y los recursos del proyecto, se seleccionaron las pymes que servirían de casos. Luego, cada empresario reunido con sus directivos y con los investigadores, eligió el proceso donde se llevaría a cabo el proyecto y firmó el acta respectiva.

Posteriormente, con el fin de desarrollar en los colaboradores involucrados en el proyecto, habilidades para identificar los principales desperdicios de manufactura, se llevó a cabo en cada empresa, una capacitación sobre: 7 desperdicios, $5 \mathrm{~S}$ y gerencia visual, la cual incorporó principios constructivistas, de aprendizaje lúdico, colaborativo y basado en problemas. En algunos casos el sitio de despliegue fue la misma empresa y en otros, el edificio de extensión de la Universidad de Antioquia. El método empleado para capacitar constó de cuatro fases: motivación, desarrollo conceptual, desarrollo procedimental y lecciones aprendidas.

En la etapa de desarrollo procedimental se utilizó la lúdica "El Avión de la Muda"® [34]; en ella, los trabajadores vivieron la experiencia de los desperdicios de manufactura en un entorno simulado de trabajo donde se ensamblan aviones de papel. En cada empresa, la capacitación tuvo una duración de cuatro horas consecutivas y fue dirigida a la totalidad de trabajadores del proceso objeto de estudio.

Una semana después de la capacitación, a través de una sesión en profundidad, se administró a los trabajadores un formulario de identificación de mudas, elaborado por el equipo y previamente adaptado al lenguaje y características de cada empresa. Al inicio de la sesión se dio un breve repaso a los conceptos vistos en la capacitación, luego se formaron grupos de trabajo, a fin de facilitar el acompañamiento de los moderadores; posteriormente se entregó el formulario a cada trabajador, para que con el debido acompañamiento, registrara los desperdicios de manufactura a los que está expuesto en su puesto de trabajo. El papel de los moderadores fue fundamental para el éxito de la sesión, garantizando que la motivación, la comprensión de los ítems y el deseo de participación se mantuviesen a lo largo de la actividad. 


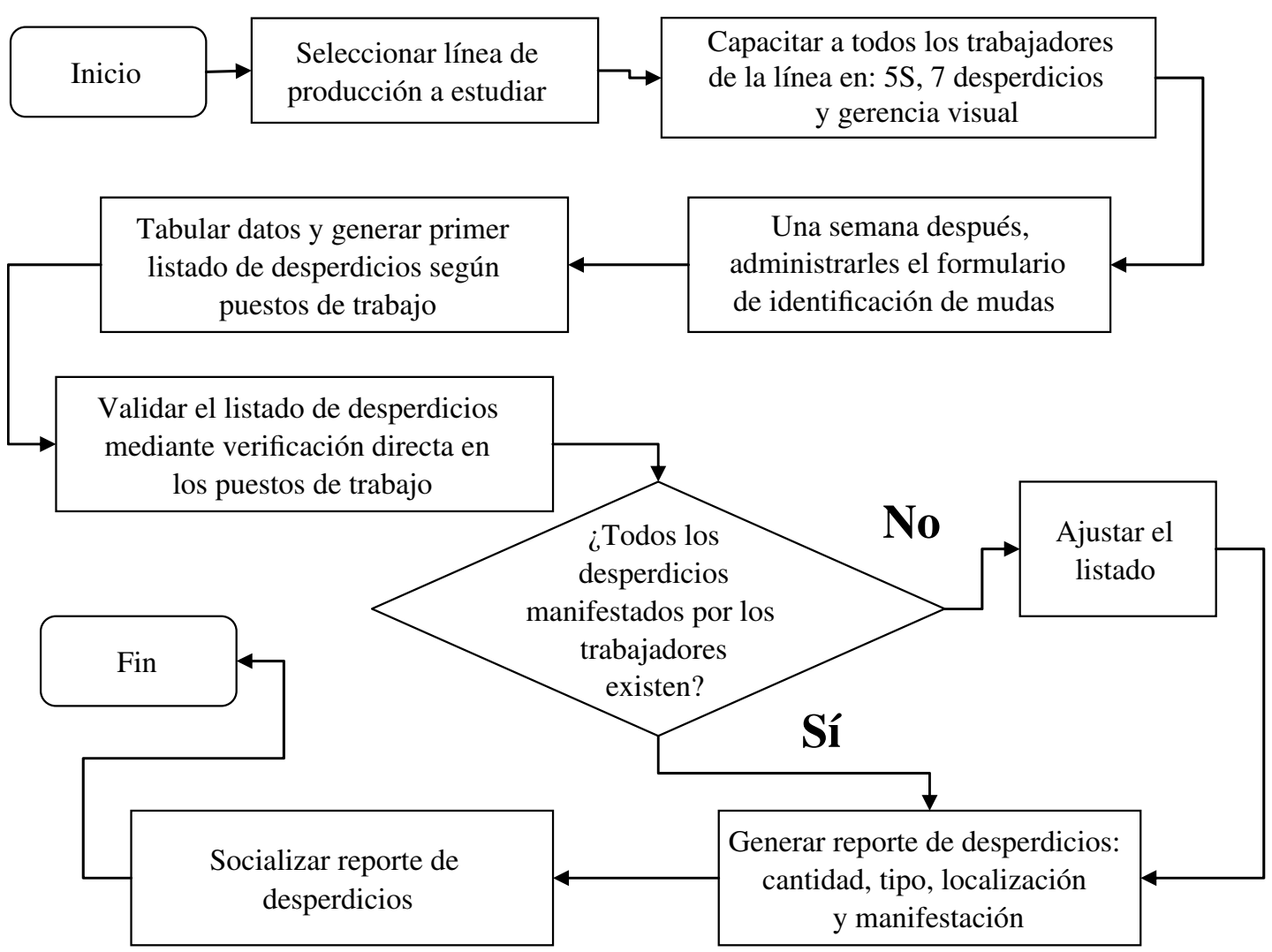

Figura 1. Procedimiento para capacitar e identificar, seguido en cada empresa.

Luego de tabulada la información, se realizó una visita a los puestos de trabajo, con el propósito de verificar la existencia de cada uno de los desperdicios de manufactura descritos por los trabajadores en el formulario. Toda la actividad tuvo una duración de entre dos y cuatro horas consecutivas, en cada empresa, dependiendo de la cantidad de puestos de trabajo.

El procedimiento culminó con la tabulación, generación de resultados, análisis, elaboración de informes para cada empresa y exposición de los mismos antes los directivos de cada compañía.

\section{RESULTADOS}

Capacitación a los trabajadores, administración del formulario y verificación en el gemba

Se capacitaron 151 empleados de las empresas objeto de estudio. En la Figura 2 se muestra un mosaico de las capacitaciones realizadas en algunas de las empresas y, en la Figura 3, se evidencia parte de las sesiones en profundidad donde se administró el formulario de identificación del muda.

Entre las lecciones aprendidas manifestadas por los trabajadores, están: "la calidad depende del trabajo en equipo", "me sentí a gusto porque aquí en este ejercicio detecté cosas innecesarias para uno y para la empresa", "la metodología utilizada despierta un enorme interés en identificación, hasta mejoramiento, en favor de eliminar mudas en el proceso y en la vida cotidiana, además de crear un apoyo en todo el equipo de trabajo", "con ideas sencillas basadas en el sentido común, podemos hacer grandes cambios en nuestra organización".

Para obtener información sobre la satisfacción de los trabajadores con la capacitación y la sesión en profundidad, se administró un cuestionario de 19 ítems, previamente validado con estudiantes, arrojando un coeficiente Alpha Cronbach de 0,91. La escala fue la Likert, con 1 (totalmente en desacuerdo) y 5 


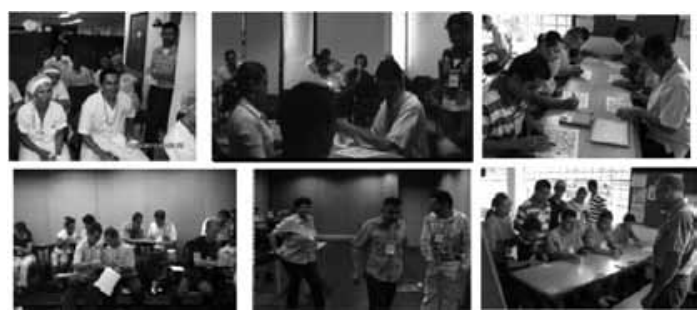

Figura 2. Algunos momentos de capacitación.
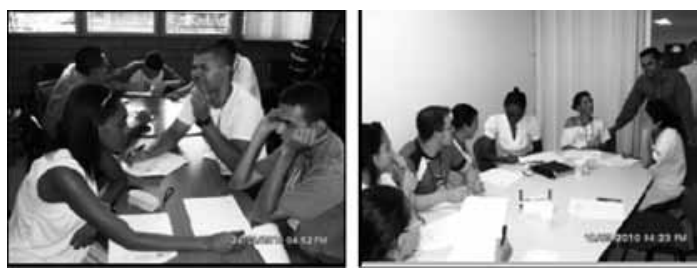

Figura 3. Administración del formulario de identificación del muda en dos de las empresas.

(totalmente de acuerdo). En todas las empresas el puntaje promedio fue superior a 4,5 .

\section{Caracterización de los principales desperdicios}

A continuación, atendiendo a criterios de extensión del manuscrito, se presenta en detalle el procedimiento seguido, pero enfocados en una de las nueve empresas, la cual se denotó por la letra "A". Ésta trata de una microempresa que posee ocho trabajadores incluyendo el gerente, y se dedica a la producción de moldes. Para las ocho empresas restantes, luego de mostrar la aplicación de dicho procedimiento, se exponen en forma consolidada los resultados.
En la Tabla 1, ilustrando para la empresa "A", respecto a las variables tipo y manifestación del muda, puede deducirse el nivel de detalle logrado, en cada firma, con el procedimiento propuesto. Del mismo modo, en la Tabla 2 se ilustra dicho detalle para la variable: localización del muda.

Consolidando los resultados para las nueve empresas, se observa en la Tabla 3 que, en promedio, la cantidad de centros de trabajo por proceso estudiado fue 6 , impactando en el desarrollo de habilidades y destrezas de 151 trabajadores operativos (media de 17 por empresa). Se encontraron en total 1.085 en las categorías: transporte, movimientos, procesos y tiempos de espera, con un promedio de 121 por proceso_empresa. En el grupo de empresas, la distribución de los desperdicios fue así: transportes $(31 \%)$, movimientos $(29 \%)$, procesos innecesarios (23\%) y tiempos de espera (18\%). A pesar de trabajar con sectores diferentes y con nueve empresas, la variación se mostró baja (entre 5,9\% y 7,2\%).

\section{DISCUSIÓN}

La metodología propuesta, que desde un punto de vista macro se resume en las etapas: capacitar e identificar, permitió descubrir y caracterizar los principales desperdicios que disminuyen la calidad, la productividad, la seguridad y la oportunidad en los procesos objeto de estudio. Por ejemplo, en la empresa "A", la distribución de los desperdicios fue: tiempos de espera (16\%), procesos innecesarios (20\%),

Tabla 1. Extracto con seis de los movimientos innecesarios en la línea de la empresa "A".

\begin{tabular}{|c|l|}
\hline Centro & \multicolumn{1}{c|}{ Descripción del $\boldsymbol{m u d a}$} \\
\hline \multirow{5}{*}{$\begin{array}{c}\text { Almacén / } \\
\text { empaque }\end{array}$} & $\begin{array}{l}\text { Debo buscar la bolsa o caja adecuada entre varias para empacar el producto. } \\
\text { Las bolsas se sacan del empaque que corresponda al tamaño requerido } \\
\text { y están en un cajón del escritorio. }\end{array}$ \\
\cline { 2 - 3 } & $\begin{array}{l}\text { Debo agacharme a buscar una referencia de la piedra solicitada, en la } \\
\text { estantería del almacén. Las más solicitadas están en la parte de abajo. }\end{array}$ \\
\cline { 2 - 3 } & $\begin{array}{l}\text { Debo agacharme para recoger las cajas de empaque que están en el suelo, } \\
\text { en el almacén de cajas. }\end{array}$ \\
\cline { 2 - 3 } Enderezado & $\begin{array}{l}\text { Debo agacharme para coger periódico de una caja que está debajo del } \\
\text { escritorio en el área de empaque para envolver el producto terminado. }\end{array}$ \\
\hline & $\begin{array}{l}\text { Girar varias veces la manila del mandril para enderezar el vástago } \\
\text { vástago, a un lado de la mesa de trabajo para enderezarlas. }\end{array}$ \\
\cline { 2 - 3 } & $\ldots$ \\
\hline
\end{tabular}


Tabla 2. Mudas según tipo y centro de trabajo para la empresa "A".

\begin{tabular}{|c|c|c|c|c|c|c|c|c|}
\hline \multirow[b]{2}{*}{$\begin{array}{c}\text { TIPO DE } \\
\text { MUDA }\end{array}$} & \multicolumn{8}{|c|}{ CENTRO DE TRABAJO } \\
\hline & $\begin{array}{c}\text { Preparación } \\
\text { de materia } \\
\text { prima }\end{array}$ & Enderezado & $\begin{array}{l}\text { Fabricación } \\
\text { de vástago }\end{array}$ & $\begin{array}{c}\text { Moldeado o } \\
\text { prensaje }\end{array}$ & Horneado & Pegado & $\begin{array}{l}\text { Almacén } \\
\text { de empaque }\end{array}$ & Total \\
\hline Espera & 1 & 0 & 1 & 5 & 4 & 0 & 3 & 14 \\
\hline $\begin{array}{l}\text { Movimientos } \\
\text { Innecesarios }\end{array}$ & 4 & 3 & 3 & 5 & 7 & 2 & 6 & 30 \\
\hline $\begin{array}{l}\text { Procesos } \\
\text { Innecesarios }\end{array}$ & 0 & 2 & 3 & 6 & 2 & 1 & 3 & 17 \\
\hline Transporte & 1 & 1 & 5 & 6 & 2 & 6 & 4 & 25 \\
\hline Total & 6 & 6 & 12 & 22 & 15 & 9 & 16 & 86 \\
\hline
\end{tabular}

Tabla 3. Consolidado del muda en las nueve empresas.

\begin{tabular}{|c|c|c|c|c|c|c|c|c|c|}
\hline \multirow[b]{2}{*}{ Empresa } & \multirow[b]{2}{*}{ Sector } & \multirow{2}{*}{$\begin{array}{c}\text { Proceso } \\
\text { estudiado }\end{array}$} & \multirow{2}{*}{$\begin{array}{c}\text { Nro. de } \\
\text { Centros } \\
\text { de trabajo } \\
\end{array}$} & \multirow{2}{*}{$\begin{array}{c}\text { Nro. } \\
\text { empleados } \\
\text { del proceso }\end{array}$} & \multirow{2}{*}{$\begin{array}{l}\text { Cantidad } \\
\text { de mudas }\end{array}$} & \multicolumn{4}{|c|}{ Distribución del $m u d a$} \\
\hline & & & & & & Espera & \begin{tabular}{|c|} 
Movimientos \\
innecesarios
\end{tabular} & $\begin{array}{c}\text { Procesos } \\
\text { innecesarios }\end{array}$ & Transporte \\
\hline A & Moldes & Puntas & 7 & 8 & 86 & $16 \%$ & $35 \%$ & $20 \%$ & $29 \%$ \\
\hline B & Metalmecánica & Pernos & 5 & 13 & 81 & $18 \%$ & $35 \%$ & $12 \%$ & $35 \%$ \\
\hline $\mathrm{C}$ & Caucho/Cuero & Extrusión & 2 & 18 & 116 & $24 \%$ & $23 \%$ & $30 \%$ & $23 \%$ \\
\hline $\mathrm{D}$ & Moldes & Ruedas & 3 & 10 & 48 & $6 \%$ & $38 \%$ & $33 \%$ & $23 \%$ \\
\hline $\mathrm{E}$ & Fundición & Mecánica & 8 & 30 & 165 & $23 \%$ & $21 \%$ & $21 \%$ & $35 \%$ \\
\hline $\mathrm{F}$ & $\begin{array}{c}\text { Estructuras } \\
\text { metálicas }\end{array}$ & Galvanizado & 8 & 22 & 122 & $21 \%$ & $24 \%$ & $17 \%$ & $38 \%$ \\
\hline $\mathrm{G}$ & $\begin{array}{c}\text { Impresión / } \\
\text { edición }\end{array}$ & Screen & 8 & 17 & 202 & $15 \%$ & $25 \%$ & $20 \%$ & $40 \%$ \\
\hline $\mathrm{H}$ & Químicos & Pintura & 6 & 14 & 148 & $14 \%$ & $30 \%$ & $23 \%$ & $33 \%$ \\
\hline I & Alimentos & $\begin{array}{l}\text { Chóloco } \\
\text { sencillo }\end{array}$ & 7 & 19 & 117 & $24 \%$ & $29 \%$ & $27 \%$ & $20 \%$ \\
\hline \multicolumn{3}{|c|}{ Totales } & 54 & 151 & 1085 & \multicolumn{4}{|c|}{ N/A } \\
\hline \multicolumn{3}{|c|}{ Promedios } & 6,0 & 16,8 & 120,6 & $18 \%$ & $\underline{29 \%}$ & $23 \%$ & $\underline{31 \%}$ \\
\hline \multicolumn{3}{|c|}{ Desviación } & 2,2 & 6,6 & 47 & $5,9 \%$ & $6,1 \%$ & $6,5 \%$ & $7,2 \%$ \\
\hline
\end{tabular}

transportes (29\%) y movimientos (35\%). Consolidando para las nueve empresas, se encontró un promedio de 121 mudas por proceso-empresa, donde el transporte y los movimientos innecesarios reunieron el $60 \%$ del muda, lo que deduce la necesidad de intervenir dichas empresas en términos de la distribución de la planta y del diseño de los puestos de trabajo, también con criterios de ergonomía.

Este estudio corrobora hallazgos de autores como [5], donde se resaltan las falencias en la comunicación y el poco valor agregado de los procesos de las pymes. La literatura enfocada en los principios del TPS presenta amplia aplicación de herramientas como el VSM, para identificar las actividades que no generan valor para el consumidor [29-31]. Esta herramienta, si bien ha evidenciado ser útil para el mejoramiento empresarial, su enfoque tiene una perspectiva macro, procurando observar toda la cadena de valor desde que se genera la solicitud del cliente hasta que se satisface la necesidad, no siendo viable descubrir, por ejemplo, el detalle de un muda de movimientos innecesarios como la inclinación de un trabajador para tomar una herramienta. Ello hace pertinente y complementario el enfoque metodológico propuesto en este manuscrito, en el sentido de que centra su 
atención en lo micro, revelando detalles mínimos de la operación en piso, ya que estudia cada centro de trabajo considerando las experiencias, opiniones, observación y conocimiento de los trabajadores, nivel operativo, previo desarrollo de habilidades y destrezas en los mismos para descubrir el muda.

En términos generales, el enfoque metodológico propuesto en este artículo está centrado en motivar, capacitar, escuchar y dar participación activa al nivel operativo, como un elemento clave para la adaptación exitosa de los principios del TPS [15, $21,24,26,32]$, considerando, como es sugerido, las particularidades de los ámbitos de aplicación [25].

\section{CONCLUSIONES}

El despliegue de la metodología propuesta posibilitó la participación activa de los trabajadores que conocen los detalles de la operación en "piso". Estos fueron capacitados mediante estrategias de aprendizaje activo, propiciando: coproducción del aprendizaje, cooperación y resolución de problemas. Todo ello, a fin de que los trabajadores pudiesen identificar los principales desperdicios de manufactura a los que están expuestos, introduciendo en sus vocablos una nueva definición para el término muda, adoptado y recordado con facilidad.

Otras evidencias de la participación activa del personal, fueron: sesión en profundidad y verificación en los puestos de trabajo, donde se les propició un escenario para ser escuchados, expresando cada uno la forma en que se manifiestan dichos desperdicios. Algunas de las lecciones aprendidas por los colaboradores, a nivel personal, fueron: "la calidad depende del trabajo en equipo", "nosotros podemos ser parte de un proceso de cambio y mejora en nuestra empresa, empezando desde nuestro sitio de trabajo y visualizando el futuro", "la importancia del trabajo en equipo, al ver que el trabajo de uno afecta positiva o negativamente el desempeño del otro".

Este estudio evidencia ruptura en la comunicación entre el nivel operativo y los demás, por lo que proporciona detalles de la operación en "piso", algunos del sentido común, con los que tradicionalmente la empresa ha convivido, generando miopía directiva hacia la reducción del muda de manufactura. Para los trabajadores, era como si tuviesen una venda que impide percibir dichas actividades como desfavorables, bajo el emblema "eso se tiene que hacer asî", pero una vez esta venda se derriba, surge una necesidad, inesperada en ellos, brotando innumerables aportes para dejar de aceptar los desperdicios.

Esta cultura, que contrae la resignación ante las actividades que no agregan valor, fue uno de los elementos más resaltados por los directivos en la primera reunión de líderes empresariales que realizó el equipo de investigación el 20 de mayo de 2010 en el edificio de extensión de la Universidad de Antioquia. Fue satisfactorio notar que la palabra muda y todo lo que en ella subyace fue interiorizado en el nivel operativo, antes familiarizado solo con el producir, obviando lo fundamental que es para el progreso de la empresa.

Aparte de los resultados analíticos de los nueve estudios de caso donde fueron implementadas las dos primeras etapas de la metodología sometida a ensayo (capacitar e identificar), se propone un enfoque novedoso, no encontrado en la revisión literaria, que coadyuva hacia la organización que aprende, y que permite no solo identificar los principales desperdicios de manufactura desde una fuente tan fidedigna como es el nivel operativo, sino también caracterizarlos en términos de: cantidad, tipo, localización y manifestación, lo cual se constituye en un posible campo de investigación para la comunidad académica.

\section{AGRADECIMIENTOS}

Los autores agradecen a las nueve empresas por el compromiso y la disposición manifestada durante el proyecto. También al Centro de Investigaciones Ambientales y de Ingeniería (CIA), a las directivas y profesores del Departamento de Ingeniería Industrial de la Universidad de Antioquia, por el apoyo y compromiso con el fortalecimiento de la investigación aplicada a la solución de problemas reales del medio empresarial.

\section{REFERENCIAS}

[1] ACOPI. Sección PYMES. En: BussinesCol. com. http://www.businesscol.com/ empresarial/pymes/ [Consulta 27-05-2010].

[2] RGS. Red de Gestores Sociales. Retrospectiva de las pequeñas y medianas empresas. Las Pymes se erigen como las principales 
promotoras del empleo en Colombia. Gobierno en línea. http://www.rgs.gov. co/noticias.shtml? $x=12916$. [Consulta 29-01-2011]

[3] M. Pérez. "Consideraciones teóricas para el análisis de las pequeñas y medianas empresas como fuente de generación de empleo". Revista Venezolana de Gerencia. Vol. 12 No.39, pp. 475-487. 2007.

[4] Latinpyme. "Habrá estímulos a pymes formales y generadoras de empleo: Santos". Fecha de consulta: 10 de enero 2011. En http://latinpymes.com/site/2010/07/ habra-estimulos-a-pymes-formales-ygeneradoras-de-empleo-santos/

[5] R. Biasca, C. Andriani y M. Rodríguez. "Un nuevo sistema de gestión para lograr PYMES de clase mundial". Editorial Norma S.A., pp. xvii-xviii. 2003.

[6] D. Sipper y R. Bulfin Jr. "Planeación y Control de la Producción". McGraw-Hill, pp. 1-7, México. 1998.

[7] P. Ruiz. "La gestión de costes en Lean manufacturing. Cómo evaluar las mejoras en costes en un sistema Lean". Netbiblo, p. 17. 2007.

[8] T. Ohno. "Toyota production system: beyond large-scale production”. Productivity Press. 1st Edition, pp. 1-143. 1988.

[9] A. Galgano y C. Nieva. "Las tres revoluciones: Caza del desperdicio: doblar la productividad con la "Lean Production". Ediciones Díaz de Santos, pp. 79-86. 2003.

[10] M. Rajadell y F. Garriga. "El control visual de la producción como fuente de ventaja competitiva”. IX Congreso de Ingeniería de Organización Gijón, 8 y 9 de septiembre 2005. Fecha de consulta: 27 mayo 2007. http://io.us. es/cio2005/items/ponencias/9.pdf

[11] T. Howell. "Actionable performance measurement: a key to success". ASQ Quality Press, p. 236. 2006.

[12] L. Codina. "Scopus: el mayor navegador científico de la Web". El Profesional de la información. Vol. 14 No. 1, pp. 44-49. 2005.

[13] T. Persoon, S. Zaleski and J. Frerichs. "Improving preanalytic processes using the principles of lean production (Toyota Production System)". American Journal of Clinical Pathology. Vol. 125 , Issue 1, pp. 16-25. 2006.
[14] S. Raab, C. Andrew, J. Condel and D. Dabbs. "Improving Papanicolaou test quality and reducing medical errors by using Toyota production system methods". American Journal of Obstetrics and Gynecology. Vol. 194, Issue 1, pp. 57-64. 2006.

[15] J. Worley and T. Doolen. "The role of communication and management support in a lean manufacturing implementation". Management Decision. Vol. 44, Issue 2, pp. 228-245. 2006.

[16] S. Taj and L. Berro. "Application of constrained management and lean manufacturing in developing best practices for productivity improvement in an auto-assembly plant". International Journal of Productivity and Performance Management. Vol. 55, Issue 3-4, pp. 332-345. 2006.

[17] D. Cumbo, D. Kline and M. Bumgardner. "Benchmarking performance measurement and lean manufacturing in the rough mill". Forest Products Journal. Vol. 56, Issue 6, pp. 25-30. 2006.

[18] H. Weller, D. Nirschl, E. Petrillo, M. Poss, C. Andres, C. Cavallaro, M. Echols, K. Grant-Young, J. Houston, A. Miller and R. Swann. "Application of lean manufacturing concepts to drug discovery: Rapid analogue library synthesis". Journal of Combinatorial Chemistry. Vol. 8, Issue 5, pp. 664-669. 2006.

[19] A. Greene and D. O'Rourke. "Lean manufacturing practice in a cGMP environment". Pharmaceutical Technology Europe. Vol. 18, Issue 10, pp. 33-39. 2006.

[20] S. Spear and H. Bowen. "Decoding the DNA of the Toyota Production System". Harvard Business Review. Vol. 77, Issue 5, pp. 97106. 1999.

[21] J. Black. "Design rules for implementing the Toyota Production System". International Journal of Production Research. Vol. 45, Issue 16, pp. 3639-3641. 2007.

[22] C. Furman and R. Caplan. "Applying the Toyota Production System: Using a patient safety alert system to reduce error. Joint Commission" Journal on Quality and Patient Safety. Vol. 33, Issue 7, pp. 376-386. 2007.

[23] D. Towill. "Exploiting the DNA of the Toyota Production System". International Journal of Production Research. Vol. 45, Issue 16, pp. 3619-3637. 2007. 
[24] S. New. "Celebrating the enigma: The continuing puzzle of the Toyota Production System". International Journal of Production Research. Vol. 45, Issue 16, pp. 3545-3554. 2007.

[25] B. Lee and H. Jo. "The mutation of the Toyota Production System: Adapting the TPS at Hyundai Motor Company". International Journal of Production Research. Vol. 45, Issue 16, pp. 3665-3679. 2007.

[26] E. Lander and J. Liker. "The Toyota Production System and art: Making highly customized and creative products the Toyota way". International Journal of Production Research. Vol. 45, Issue 16, pp. 3681-3698. 2007.

[27] H. Sakai and K. Amasaka. "Development of a robot control method for curved seal extrusion for high productivity in an advanced Toyota Production System". International Journal of Computer Integrated Manufacturing. Vol. 20, Issue 5, pp. 486-496. 2007.

[28] T. Pardi. "Redefining the Toyota Production System: The European side of the story". New Technology, Work and Employment. Vol. 22, Issue 1, pp. 2-20. 2007.

[29] F. Abdulmalek and J. Rajgopal. "Analyzing the benefits of lean manufacturing and value stream mapping via simulation: A process sector case study". International Journal of Production Economics. Vol. 107, Issue 1, pp. 223-236. 2007.

[30] Y. Lian and H. Van Landeghem. "Analysing the effects of Lean manufacturing using a value stream mapping-based simulation generator". International Journal of Production Research. Vol. 45, Issue 13, pp. 3037-3058. 2007.

[31] C. Grewal. "An initiative to implement lean manufacturing using value stream mapping in a small company". International Journal of Manufacturing Technology and Management. Vol. 15, Issue 3-4, pp. 404-417. 2008.

[32] E. Dickson, S. Singh, D. Cheung, C. Wyatt and A. Nugent. "Application of Lean Manufacturing Techniques in the Emergency Department". Journal of Emergency Medicine. Vol. 37, Issue 2, pp. 177-182. 2009.

[33] G. Anand and R. Kodali. "Selection of lean manufacturing systems using the analytic network process - A case study". Journal of Manufacturing Technology Management. Vol. 20, Issue 2, pp. 258-289. 2009.

[34] J. Pérez. "El avión de la muda: herramienta de apoyo a la enseñanza-aprendizaje práctico de la manufactura esbelta”. Revista Facultad de Ingeniería de la Universidad de Antioquia. Colombia. N. 58, pp. 173-182. Marzo 2011. 